\title{
Estimating risk curves for first-degree relatives of patients with Alzheimer's disease: The REVEAL study
}

\author{
L. Adrienne Cupples, $P h D^{1,2}$, Lindsay A. Farrer, $P h D^{1-5}$, A. Dessa Sadovnick, $P h D^{6}$, Norman Relkin, $M D, P h D^{7}$,
} Peter Whitehouse, $M D, P h D^{8}$, and Robert C. Green, $M D, M P H^{2-4}$

\begin{abstract}
Purpose: The REVEAL study is a randomized, controlled study of the psychological and behavioral impact of APOE disclosure in a risk assessment protocol provided to first degree relatives of patients with Alzheimer's disease. The protocol presents risk information as cumulative incidence curves. This article describes how these curves were estimated. Methods: Curves were calculated using Bayes' rule to compute the posterior survival curves incorporating APOE information. Results: A combination of survival data from the MIRAGE study and gender- and age-specific APOE odds ratios were used to create risk curves for males and females within each of the 6 APOE genotypes. Conclusion: Utilizing comparative genotype relative risk information and survival data from family studies, estimates of gender-, age-, and genotype-specific risk can be generated for use in a risk assessment research study that features genotype disclosure. Genet Med 2004:6(4):192-196.
\end{abstract}

Key Words: risk prediction, genetic counseling, Alzheimer's disease

Alzheimer's disease (AD) is the leading cause of cognitive impairment among older persons, ${ }^{1}$ and in the U.S., the prevalence of $\mathrm{AD}$ will continue to increase with the aging population. ${ }^{2}$ Although the etiology of $\mathrm{AD}$ is not fully understood, there is clear evidence that genetic factors are important determinants of AD., ${ }^{3,4}$ The gene for apolipoprotein $\mathrm{E}$ (APOE) has been identified as a common susceptibility polymorphism ${ }^{5}$ with allele $\epsilon 4$ conferring 5 to 15 times greater risk, depending upon whether an individual has one or two copies. ${ }^{6}$ The $\epsilon 4$ allele is common in the U.S. population with a frequency of about $14 \%$. Although individuals with one or two $\epsilon 4$ alleles can attain late age without developing $\mathrm{AD}$, the burden of risk attributable to APOE is substantial. ${ }^{7}$

Relatives of individuals affected with $\mathrm{AD}$ are increasingly aware that they may be at increased risk for developing $\mathrm{AD}$ themselves. Surveys and data from this study have demonstrated that substantial portions of such relatives, often over half of respondents, are interested in more fully understanding their risk. ${ }^{7-11}$ The Risk Evaluation and Education for Alzheimer's Disease (REVEAL) study was established to evaluate the feasibility, safety, and behavioral responses of providing gen-

From the Departments of ${ }^{1}$ Biostatistics and ${ }^{2}$ Epidemiology, Boston University School of Public Health, Boston, Massachusetts; Departments of ${ }^{3}$ Medicine (Genetics Program), ${ }^{4}$ Neurology, and ${ }^{5}$ Genetics \& Genomics, Boston University School of Medicine, Boston, Massachusetts; ${ }^{6}$ Department of Medical Genetics and Faculty of Medicine, Division of Neurology, University of British Columbia, Vancouver, Canada; ${ }^{7}$ Department of Neurology, Weill Medical College of Cornell University, New York; ${ }^{8}$ Memory and Aging Center, Case Western Reserve University/University Hospitals of Cleveland, Cleveland, Ohio.

L. Adrienne Cupples, Department of Biostatistics, Boston University School of Public Health, 715 Albany Street, Boston, MA 02118

Received: December 23, 2004.

Accepted: February 25, 2004.

DOI: 10.1097/01.GIM.0000132679.92238.58 eral information on risk and specific risk assessment, including APOE genotype disclosure, to adult children of patients with $\mathrm{AD}$ (the "subjects" of our study). ${ }^{12}$ These subjects were recruited either through systematic ascertainment from AD research registries at Boston University, Case Western, and Cornell or through self-referral at each site. After a general information session and administration of baseline questionnaires, subjects were randomly assigned to either the "Control" group that would receive risk information based on age, gender, and family history only or the "Intervention" group that would receive the same risk information as the controls plus risk information based on the subject's APOE genotype. After disclosure of the risk information by a genetic counselor, the Control and Intervention groups were followed for one year through telephone and in-person structured interviews conducted by genetic counselors. The two groups were compared on a range of psychological responses and behavioral changes, including evaluation within each group of changes from baseline.

For the risk disclosure portion of the REVEAL study, risk curves were created, incorporating gender, age, family history, and for the Intervention group, APOE genotype. Two sets of risk curves were developed: one set for those in the Intervention group, and another set for those in the Control group. The purpose of this article is to describe the methods used to construct these curves.

\section{MATERIALS AND METHODS}

To create the risk curves for the REVEAL study participants, we drew upon previously published estimates of gender- and age-specific family risk, including the findings over the past 11 years from the Multi-Institutional Research in Alzheimer's Ge- 
netic Epidemiology (MIRAGE) study. ${ }^{4,6,13-15}$ Specifically, we used two sources of information to develop these risk curves: (1) gender- and age-specific incidence curves for first-degree relatives of persons with $\mathrm{AD}$ (comparable to those previously published in Green et al., ${ }^{4}$ Cupples et al., ${ }^{14}$ and Lautenschlager et al. ${ }^{15}$ ). and (2) APOE genotype-specific odds ratio estimates for each gender and age reported in a meta analysis of data from over 50 studies worldwide. ${ }^{6}$ The odds ratio estimates used in this manuscript are based on clinic studies of Caucasians. We found these estimates to be homogeneous across these types of studies of this ethnic group. In this section we describe how we used these two sources of information to derive risk curve estimates for REVEAL study subjects.

Control risk curves were based on information from a sample of 12,630 first-degree relatives (6,236 males, 6,394 females) and 1853 spouses. These curves were nearly identical to those reported by Lautenschlager et al. ${ }^{15}$ To incorporate APOE genotype information for the Intervention group, we used Bayes rule that states the posterior probability of an event can be calculated from the prior probability. ${ }^{16-17}$ Specifically, if $A$ and $\mathrm{B}$ were two events or two traits, then the following:

$$
\operatorname{Pr}(\mathrm{A} \mid \mathrm{B})=\frac{\operatorname{Pr}(\mathrm{A}) \operatorname{Pr}(\mathrm{B} \mid \mathrm{A})}{\operatorname{Pr}(\mathrm{B})},
$$

where $\operatorname{Pr}(\mathrm{A} \mid \mathrm{B})$ was the posterior probability of A given $\mathrm{B}(\mathrm{A}$ among those with trait $B$ ) and the prior probability was $\operatorname{Pr}(A)$. An analogous equation can be written for the $\operatorname{Pr}($ not $A \mid B)=\operatorname{Pr}$ $(\overline{\mathrm{A}} \mid \mathrm{B})$ as follows:

$$
\operatorname{Pr}(\overline{\mathrm{A}} \mid \mathrm{B})=\frac{\operatorname{Pr}(\overline{\mathrm{A}}) \operatorname{Pr}(\mathrm{B} \mid \overline{\mathrm{A}})}{\operatorname{Pr}(\mathrm{B})}
$$

Using these two equations, we calculated the posterior odds, the ratio of the probability of an event to the probability of its complement. Hence, the posterior odds of A given B was as follows:

$$
\begin{aligned}
\text { Posterior } \operatorname{Odds}(\mathrm{A} \mid \mathrm{B})=\frac{\operatorname{Pr}(\mathrm{A} \mid \mathrm{B})}{\operatorname{Pr}(\overline{\mathrm{A}} \mid \mathrm{B})}= & \frac{\operatorname{Pr}(\mathrm{A}) \operatorname{Pr}(\mathrm{B} \mid \mathrm{A})}{\operatorname{Pr}(\overline{\mathrm{A}}) \operatorname{Pr}(\mathrm{B} \mid \overline{\mathrm{A}})} \\
& =\operatorname{Prior} \operatorname{Odds}(\mathrm{A}) \frac{\operatorname{Pr}(\mathrm{B} \mid \mathrm{A})}{\operatorname{Pr}(\mathrm{B} \mid \overline{\mathrm{A}})}
\end{aligned}
$$

Note that the term on the far right is the relative risk of $\mathrm{B}$ comparing those with A to those without A. This relative risk can be estimated by an odds ratio. Hence, we had the following:

$$
\operatorname{Posterior} \operatorname{Odds}(\mathrm{A} \mid \mathrm{B})=\operatorname{Prior} \operatorname{Odds}(\mathrm{A}) \mathrm{RR}(\mathrm{B}) \text {, }
$$

where the $R R(B)$ was understood to be the risk of $B$ in those with A compared to that of those without A.

In a logistic model, we can state the probability of an event $\mathrm{E}$ as a function of known covariates, $\mathrm{X}_{1}, \mathrm{X}_{2}, \ldots, \mathrm{X}_{\mathrm{k}}$, as follows:

$$
p=\operatorname{Pr}\left(E \mid X_{1}, X_{2}, \ldots, X_{k}\right)=\frac{e^{b_{0}+b_{1} X_{1}+b_{2} X_{2}+\ldots+b_{k} X_{k}}}{1+e^{b_{0}+b_{1} X_{1}+b_{2} X_{2}+\ldots+b_{k} X_{k}}}
$$

or

$$
\text { Odds }=\frac{p}{1-p}=e^{b_{0}+b_{1} X_{1}+b_{2} X_{2}+\ldots+b_{k} X_{k}}
$$

Therefore,

$$
\mathrm{p}=\operatorname{Pr}\left(\mathrm{E} \mid \mathrm{X}_{1}, \mathrm{X}_{2}, \ldots, \mathrm{X}_{\mathrm{k}}\right)=\frac{\text { Odds }}{1+\text { Odds }}
$$

Combining this information, we expressed the posterior probability as follows:

$$
\begin{aligned}
\text { posterior } \mathrm{p}=\operatorname{Pr}\left(\mathrm{E} \mid \mathrm{X}_{1}, \mathrm{X}_{2}, \mathrm{~K}, \mathrm{X}_{\mathrm{k}}\right)=\frac{\text { Posterior Odds }}{1+\text { Posterior Odds }} \\
=\frac{(\text { Prior Odds }) \mathrm{RR}}{1+(\text { Prior Odds }) \mathrm{RR}}
\end{aligned}
$$

Applying this derivation to REVEAL, we let A in the description above be the event that $\mathrm{AD}$ occurs by age $a(\mathrm{AD} \leq a)$ and $\mathrm{B}$ be the APOE genotype. According to this derivation, we thus had the following:

$$
\begin{aligned}
& \text { posterior } \mathrm{p}(\mathrm{AD} \leq \mathrm{a} \mid \mathrm{APOE}) \\
& =\frac{\text { Posterior Odds }(\mathrm{AD} \mid \text { age } \mathrm{a}, \mathrm{APOE}=\mathrm{g})}{1+\text { Posterior Odds }(\mathrm{AD} \mid \text { age } \mathrm{a}, \mathrm{APOE}=\mathrm{g})} \\
& =\frac{[\text { Prior Odds }(\mathrm{AD} \mid \text { age } \mathrm{a})] \mathrm{RR}(\mathrm{APOE}=\mathrm{g} \mid \mathrm{AD} \leq \mathrm{a})}{1+[\text { Prior Odds }(\mathrm{AD} \mid \text { age } \mathrm{a})] \mathrm{RR}(\mathrm{APOE}=\mathrm{g} \mid \mathrm{AD} \leq \mathrm{a})}
\end{aligned}
$$

Because we did not have reliable information on the $\mathrm{RR}(\mathrm{APOE}=\mathrm{g} \mid \mathrm{AD} \leq a)$, we proposed to estimate the relative risk by gender- and age-specific odds ratio estimates and then noted the following:

$$
\text { OR }(\text { E for } A \text { vs } \bar{A})=\operatorname{OR}(A \text { for } E \text { vs } \bar{E})
$$

Hence, we estimated the RR as follows:

$$
\begin{aligned}
& \mathrm{RR}(\mathrm{APOE}=\mathrm{g} \mid \mathrm{AD} \leq \mathrm{a} \text { vs } \mathrm{AD}>\mathrm{a}) \\
& =\frac{\operatorname{Risk}(\mathrm{APOE}=\mathrm{g} \mid \mathrm{AD} \leq \mathrm{a})}{\operatorname{Risk}(\mathrm{APOE}=\mathrm{g} \mid \mathrm{AD}>\mathrm{a})} \approx \\
& \begin{aligned}
& \mathrm{OR}(\mathrm{APOE}=\mathrm{g} \mid \mathrm{AD} \leq \mathrm{a} v \mathrm{AD}>\mathrm{a}) \\
&= \frac{\mathrm{Odds}(\mathrm{APOE}=\mathrm{g} \text { vs } \mathrm{APOE}=33 \mid \mathrm{AD} \leq \mathrm{a})}{\mathrm{Odds}(\mathrm{APOE}=\mathrm{g} \text { vs } \mathrm{APOE}=33 \mid \mathrm{AD}>\mathrm{a})} \\
&= \frac{\operatorname{Odds}(\mathrm{AD} \leq \mathrm{a} \mid \mathrm{APOE}=\mathrm{g})}{\mathrm{Odds}(\mathrm{AD} \leq \mathrm{a} \mid \mathrm{APOE}=33)} \\
&= \mathrm{OR}(\mathrm{AD} \leq \mathrm{a} \mid \mathrm{APOE}=\mathrm{g} \text { vs APOE }=33)
\end{aligned}
\end{aligned}
$$

Thus, we had the following calculation to obtain the estimated risk of AD at age $a$ for someone with APOE genotype $g$. Note that the odds ratios are gender- and age-specific, with differing estimates for each gender and each age.

posterior $\mathrm{p}(\mathrm{AD} \leq \mathrm{a} \mid \mathrm{APOE})$

$$
\begin{gathered}
{[\text { Prior Odds }(\mathrm{AD} \mid \text { age } \mathrm{a})]} \\
\mathrm{OR}(\mathrm{AD} \leq \mathrm{a} \mid \mathrm{APOE}=\mathrm{g} \text { vs APOE }=33) \\
1+[\text { Prior Odds }(\mathrm{AD} \mid \text { age } \mathrm{a})] \\
\mathrm{OR}(\mathrm{AD} \leq \mathrm{a} \mid \mathrm{APOE}=\mathrm{g} \text { vs APOE }=33)
\end{gathered}
$$




\section{Cupples et al.}

For the REVEAL study, we estimated the prior odds using the age-specific risk curve estimates derived from the MIRAGE dataset, similar to those described by Lautenschlager et al. ${ }^{15}$ and the gender-, age-, and genotype-specific odds ratio estimates calculated from the meta analysis data. ${ }^{6}$ The estimates used in this study are those drawn from clinic studies of Caucasians, which were found to be homogeneous across the studies of this ethnic group.

To provide a sense of the range of values for these estimates, we also calculated $95 \%$ confidence intervals. Standard errors for the risk curves were estimated by applying delta theorems, which state that the variance of a function of estimates can be calculated from a function of the variances and covariances of the estimates. ${ }^{18}$ Because we were considering the product and ratio of estimates, we transformed the estimator of risk to a logarithm scale to obtain a more symmetrical distribution and estimated the standard errors on the logarithm scale. Once a $95 \%$ confidence interval is calculated on the logarithm scale, the $95 \%$ confidence interval for the estimate can be readily obtained by exponentiating the limits on the logarithm scale. The derivation of the standard errors is provided in the Appendix.

\section{RESULTS}

\section{Example: Practical application for male participants in the REVEAL study}

We illustrate these methods for men in the REVEAL study. First, we calculated the risk estimates for men who are first degree relatives of an individual with $\mathrm{AD}$, using an extension of Kaplan-Meier survival methods, as described in Cupples et al. ${ }^{14}$ The primary difference between this approach and a traditional Kaplan-Meier approach is that it incorporates information from persons with missing age information (e.g., those affected with $\mathrm{AD}$, but their age at onset is unknown). The resulting age-specific risk curve is presented in Fig. 1. As a comparison for REVEAL study participants, we also estimated an

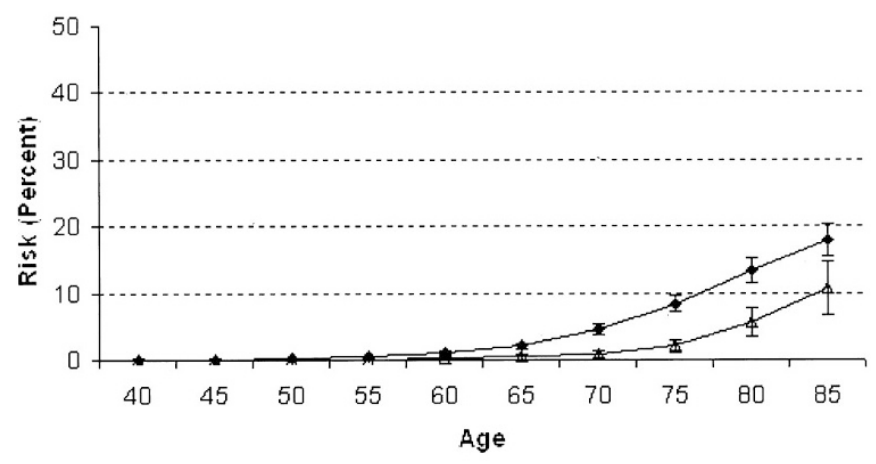

First Degree Relative $₫$ General Population

Fig. 1. Risk curves of AD for Control men. Curves are presented for ages 40 to 85 for the general population, denoted with an open triangle, and for first degree relatives of an $\mathrm{AD}$ patient, denoted with a solid diamond. Estimates of risk are given in percent. Bars represent the $95 \%$ confidence interval for each age at 5 -year intervals. age-specific risk curve for spouses of $\mathrm{AD}$ patients to use as an estimate of the risk in the general population.

The plot in Fig. 1 was used to convey information on risk of $\mathrm{AD}$ for men in the REVEAL study who were assigned to the Control group. For example, if a male entered the study at age 55 , we would tell him that his risk for $\mathrm{AD}$ up to his current age is $0.43 \%$, while by age 80 the absolute risk (risk from birth to age 80 ) is about $13.3 \%$. We could also cite his remaining risk to develop $\mathrm{AD}$ by age 80 , given that he is currently age 55 . This risk is calculated as the risk between age 55 and age 80 (risk age 80 - risk age 55) divided by the risk of developing $\mathrm{AD}$ after age 55. For this man, this risk would be as follows: $(13.3-0.43) /$ $(100-0.43)=12.9 \%$

Similarly, risk estimates could be provided for males at any other age. Using the example of a 65 -year old male participant in the REVEAL study, it can be calculated that the risk up until his current age of 65 was $2.0 \%$ and his absolute risk at age 80 is still $13.3 \%$. However, his remaining risk from age 65 to age 80 is $(13.3-2.0) /(100-2.0)=11.5 \%$. These calculations illustrate the fact that the risk curves present the risk from birth. As individuals age without developing $\mathrm{AD}$, their remaining risk actually decreases, since they have passed through ages when they could have developed AD but did not.

For male participants in the REVEAL study Intervention group, we incorporated the gender- and age-specific risk based on their APOE genotype, as illustrated in Fig. 2 for men with APOE genotype $\epsilon 3 / \epsilon 4$. This curve was calculated according to the methods described earlier. To create the curve, we modified the age-specific risk estimates for men who are first-degree relatives of an individual with $\mathrm{AD}$ by the age-specific odds ratio estimates for a male with APOE genotype $\epsilon 3 / \epsilon 4$ relative to a male with APOE genotype $\epsilon 3 / \epsilon 3$. For example, in our metaanalysis article, we found that the odds ratio estimate for a 55 -year-old Caucasian male with APOE genotype $\epsilon 3 / \epsilon 4$ is 3.02, for a 65 -year-old Caucasian male with genotype $\epsilon 3 / \epsilon 4$ is 3.31 , and for an 80-year-old male is 2.37 . Hence, for a male with

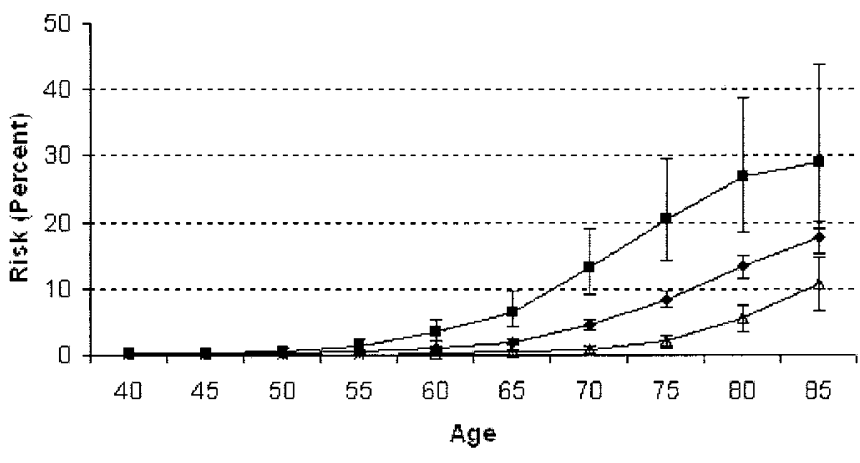

- APOE $34 \rightarrow$ General Population $\multimap-$ First Degree

Fig. 2. Risk curves of $A D$ for Intervention men with $A P O E$ genotype $\epsilon 3 / \epsilon 4$. Three curves are presented for ages 40 to 85 : one for the general population, denoted with an open triangle, one for first degree male relatives of an $\mathrm{AD}$ patient, denoted with a solid diamond, and one for a first degree male relative with APOE genotype $\epsilon 3 / \epsilon 4$, denoted with a solid square. Estimates of risk are given in percent. Bars represent the $95 \%$ confidence interval for each age at 5 -year intervals. 
APOE genotype $\epsilon 3 / \epsilon 4$ and age 55 at entry into the study, the estimated risk of developing $\mathrm{AD}$ by his current age is $1.3 \%$ using equation 1 . This is calculated from the prior risk of $0.43 \%$ for a male with $\epsilon 3 / \epsilon 3$ genotype and the increased odds for the male age 55 with genotype $\epsilon 3 / \epsilon 4$ of 3.02 . The prior risk of 0.00433 is first converted to a prior odds resulting in 0.00435 . This estimate is then multiplied by the odds ratio as 0.00435 (3.02) yielding 0.0131 . Finally, the risk for the male age 55 with genotype $\epsilon 3 / \epsilon 4$ is calculated as $0.0131 /(1+0.0131)$ yielding 0.01297 . His absolute risk of developing $\mathrm{AD}$ by age 80 is $26.8 \%$, using the risk of 0.13344 for the male age 80 with genotype $\epsilon 3 / \epsilon 3$ and odds ratio 2.37 for a male age 80 with genotype $\epsilon 3 / \epsilon 4$. Thus, his remaining risk to develop AD by age 80 , given that he is currently 55 , is $25.8 \%$. Similarly, if he is age 65 with prior risk of $2.01 \%$ and odds ratio of 3.31 , his remaining risk to develop $\mathrm{AD}$ by age 80 given that he is currently 65 (current risk of 6.4\%) is about $21.8 \%$. Fig. 2 presents the confidence limits for each curve along with the estimated curves.

Thus, to illustrate, during disclosure by the genetic counselor, a male REVEAL participant in the Intervention group with APOE $\epsilon 3 / \epsilon 4$ would be shown the curves presented in Fig. 2, whereas a male REVEAL participant in the Control group would be shown the curves in Fig. 1.

In this study, we have presented the methods for males and for males with APOE $\epsilon 3 / \epsilon 4$. The methods for other groups of individuals are comparable. Curves for these groups are available from the authors.

\section{DISCUSSION}

It has been firmly established that the $\epsilon 4$ allele of APOE carries substantial increased risk for $\mathrm{AD}$. In light of the physical, psychological, and social burdens of $\mathrm{AD}$, adult children having one parent with $\mathrm{AD}$ are increasingly concerned about their own risk of developing AD. Many are aware of APOE as a genetic risk factor and realize that there is a potential predictive genetic test for $\mathrm{AD}$. Some actually request information about their risks including APOE genotype information, from clinicians. However, there have been several consensus groups that have recommended against disclosure of APOE genotype to unaffected persons, and there are no data on this to help guide clinical protocols.

In response to this situation, a multicenter study called the REVEAL study (Risk Evaluation and Education for Alzheimer's disease) was funded in 1999 by the Ethical, Legal, and Social Implications program of the National Human Genome Research Institute. A primary goal of this study was to evaluate the safety of presenting risk information to offspring of a person with AD. For this goal, the REVEAL study protocol randomized adult offspring of $\mathrm{AD}$ patients into one of two groups: one group received information about their risk based on family history and their APOE genotype (the Intervention group), and a second group received information about their risk based on family history only (the Control group). To facilitate the disclosure of risks to Intervention and Control groups, we decided to develop risk curves that illustrated the information in a manner comprehensible to study participants. This article describes the methods used to develop these risk curves and presents some specific examples of these curves.

These risk estimates pertain only to first-degree relatives of $\mathrm{AD}$ patients. They do not allow inference about the risk (with or without APOE information) for other categories of biological relatives or for the general population. Participants in the Control Arm of the REVEAL study were presented with two curves representing the risk to a first-degree relative with the same gender as the participant, and the risk to an unrelated individual (i.e., a spouse) independent of APOE genotype. Participants in the Intervention Arm of the study were presented with the same two curves, plus a third curve, which represented the risk for a first degree relative of the same gender as the participant, incorporating his or her APOE genotype and the age-specific odds ratio estimates associated with this genotype. No confidence limits were included on the curves presented to study participants. The presentation of curves was felt to be important because it provided risk information in a graphical way that could be readily comprehended by study participants.

There are several caveats regarding the estimation of these curves. These curves were derived from two sources of information, using very different strategies for collection of data and cannot be considered definitive estimates of risk. Nevertheless, both sources of information were derived from large studies, using information from multiple institutions, both published and unpublished information, and from well-characterized individuals with AD. Further, we did not use a single odds ratio estimate. Rather, the odds ratio estimates were gender- and age-specific. An additional limitation is that the reliability of the information diminishes after age 85 because each of the original studies used to estimate risks have fewer individuals of advanced age. However, most persons seeking genetic information are middle-aged and are less concerned about their risk at these late ages. In developing this methodology, the estimates of gender- and age-specific odds ratios have been assumed to approximate risk ratios. This assumption is appropriate in several situations, but may not provide valid estimates for large risk estimates as the approximation is only accurate for rare events. Finally, our estimates were derived only for Caucasian subjects because we did not have sufficient information to derive estimates for other ethnic groups. Additional data are being collected that will eventually allow the estimation of curves specific for African Americans as well.

In summary, we describe the methods used to derive risk estimates of $\mathrm{AD}$ for offspring of $\mathrm{AD}$ patients in the context of the REVEAL study clinical trial. These methods use two sources of information including familial risk data from the MIRAGE study and results from a pooled data analysis providing estimates of the APOE gender-, age-, and genotype-specific odds of $\mathrm{AD}$. In the future, we plan to extend these methods to other ethnic groups. Ultimately, the derivation of more precise unbiased estimates will rely on prospective studies that follow high-risk individuals over a long period of time. ${ }^{12}$ 


\section{APPENDIX}

Because the posterior probability of developing AD by age $a$ is estimated by

posterior $\mathrm{p}(\mathrm{AD} \leq \mathrm{a} \mid \mathrm{APOE})$

$$
\begin{aligned}
& \text { [Prior Odds }(\mathrm{AD} \mid \text { age a })] \\
& =\frac{\mathrm{OR}(\mathrm{AD} \leq \mathrm{a} \mid \mathrm{APOE}=\mathrm{g} \text { vs } \mathrm{APOE}=33)}{1+[\text { Prior Odds }(\mathrm{AD} \mid \text { age } \mathrm{a})]} \\
& \mathrm{OR}(\mathrm{AD} \leq \mathrm{a} \mid \mathrm{APOE}=\mathrm{g} \text { vs } \mathrm{APOE}=33)
\end{aligned}
$$

and is a function of the prior odds of AD by age $a$ and the relative odds of $\mathrm{AD}$ by age $a$ for someone with APOE genotype $g$ compared to someone with APOE genotype 33, we applied delta theorems to calculate its standard error. As the distribution of this estimate may be skewed, we first applied a logarithm (base e) transformation and then calculated the standard error on the logarithmic scale. Hence, a confidence interval for the posterior risk of $\mathrm{AD}$ can be calculated on a logarithmic scale, using the derived standard error, and then each endpoint of the interval exponentiated to obtain the confidence interval in the original scale.

To obtain a formulation for the variance of the posterior risk, let the following be true: $\mathrm{p}=$ posterior $\mathrm{p}(\mathrm{AD} \leq a \mid \mathrm{APOE}) ; \xi=$ prior odds $(\mathrm{AD} \mid$ age $a) ; \mathrm{OR}=\mathrm{OR}(\mathrm{AD} \leq a \mid \mathrm{APOE}=g \mathrm{vs} . \mathrm{APOE}=33)$

Then, we see the following:

$\ln \mathrm{p}=\ln (\xi \mathrm{OR})-\ln (1+\xi \mathrm{OR})$

$$
=\ln \xi+\ln \mathrm{OR}-\ln (1+\xi \mathrm{OR})
$$

From this formulation, we see the following:

$$
\begin{aligned}
& \operatorname{Var}[\ln \mathrm{p}]= \\
& \operatorname{Var}[\ln \xi]+\operatorname{Var}[\ln \mathrm{OR}]+\operatorname{Var}[\ln (1+\xi \mathrm{OR})] \\
& +2 \operatorname{Cov}[\ln \xi, \ln \mathrm{OR}]-2 \operatorname{Cov}[\ln \xi, \ln (1+\xi \mathrm{OR})] \\
& -2 \operatorname{Cov}[\ln \mathrm{OR}, \ln (1+\xi \mathrm{OR})]
\end{aligned}
$$

Because OR $=e^{\ln O R}$, it is clear that logarithm of the posterior risk is a function of two quantities, the logarithm of the prior odds $(\ln \xi)$ and the logarithm of OR (ln OR). Thus, applying the delta theorem for the variance of two estimators and assuming that the covariance between $\ln \mathrm{OR}$ and $\xi$ is zero because these are estimated from independent data sets, we see the following:

$$
\begin{aligned}
& \operatorname{Var}[\ln \mathrm{p}] \\
& =\left(\frac{1}{\xi}\right)^{2} \operatorname{Var}[\xi]+\operatorname{Var}[\operatorname{lnOR}]+\left(\frac{\mathrm{OR}}{1+\xi \mathrm{OR}}\right)^{2} \operatorname{Var}[\xi] \\
& +\left(\frac{\xi \mathrm{OR}}{1+\xi \mathrm{OR}}\right)^{2} \operatorname{Var}[\operatorname{lnOR}]-2\left(\frac{\mathrm{OR}}{\xi(1+\xi \mathrm{OR})}\right) \operatorname{Var}[\xi] \\
& -2\left(\frac{\xi \mathrm{OR}}{(1+\xi \mathrm{OR})}\right) \operatorname{Var}[\operatorname{lnOR}] \\
& =\left[\frac{1}{\xi^{2}}+\left(\frac{\mathrm{OR}}{1+\xi \mathrm{OR}}\right)^{2}-2\left(\frac{\mathrm{OR}}{\xi(1+\xi \mathrm{OR})}\right)\right] \operatorname{Var}(\xi) \\
& +\left[1+\left(\frac{\xi \mathrm{OR}}{1+\xi \mathrm{OR}}\right)^{2}-2\left(\frac{\xi \mathrm{OR}}{(1+\xi \mathrm{OR})}\right)\right] \operatorname{Var}(\operatorname{lnOR})
\end{aligned}
$$

With this formulation, we only need to calculate the variance of two quantities, the prior odds and the ln OR. The prior odds is of the form $\mathrm{p} / 1-\mathrm{p}$ and thus,

$$
\operatorname{Var}(\xi)=\frac{1}{(1-p)^{4}} \operatorname{Var}(p)
$$

where $\mathrm{p}=$ prior odds and the $\operatorname{Var}(\ln \mathrm{OR})$ is calculated from the variance of a linear function of the logistic regression parameters from which the $\ln \mathrm{OR}$ is estimated.

\section{ACKNOWLEDGMENTS}

This research was supported by National Institutes of Health grants R01 HG/AG02213 (The REVEAL study), R01 AG09029 (The MIRAGE study), and P30 AG13846 (Boston University Alzheimer's Disease Center), a grant to the Boston University General Clinical Research Center (M01 RR00533), and a Merit Award from the Veterans Administration.

\section{References}

1. Green RC. Diagnosis and treatment of Alzheimer's disease and other dementias. Caddo, OK: Professional Communications; 2001.

2. Brookmeyer R, Gray S, Kawas C. Projections of Alzheimer's disease in the United States and the public health impact of delaying disease onset. Am J Pub Health 1998;88:1337-1342.

3. Farrer LA. Genetics and the dementia patient. The Neurologist 1997;3:13-30.

4. Green RC, Cupples LA, Go R, Benke KS, Edeki T, Griffith PA et al for the MIRAGE Study Group. Risk of Dementia among White and African American relatives of patients with Alzheimer's disease. JAMA 2002;287:329-336.

5. Corder EH, Saunders AM, Strittmatter WJ, Schmechel DE, Gaskell PC, Small GW et al. Gene dose of apolipoprotein E type 4 allele and the risk of Alzheimer's disease in late-onset families. Science 1993;261:921-923.

6. Farrer LA, Cupples LA, Haines JL, Hyman B, Kukull WA, Mayeux R et al, APOE and Alzheimer Disease Meta Analysis Consortium. Effects of age, gender and ethnicity on the association between apolipoprotein E genotype and Alzheimer disease. JAMA 1997;278:1349-1356.

7. Seshadri S, Drachman DA, Lippa CF. Apolipoprotein E epsilon 4 allele and the lifetime risk of Alzheimer's disease: What physicians know, and what they should know. Arch Neurol 1995;52:1074-1079.

8. Green R, Clarke V, Thompson V, Woodard JL, Letz R. Early detection of Alzheimer's disease: Methods, markers and misgivings. Alzheimer Dis Assoc Disord 1997; $11: S 1-S 5$.

9. Roberts JS. Anticipating response to predictive genetic testing for Alzheimer's disease: A survey of first-degree relatives. Gerontologist 2000;40:43-52.

10. Roberts JS, Connell CM. Illness representations among first-degree relatives of people with Alzheimer disease. Alzheimer Dis Assoc Disord 2000;14:129-136.

11. Roberts JS, Barber M, Brown TM, Cupples LA, Farrer LA, LaRusse SA, et al. Who seeks genetic susceptibility testing for Alzheimer's disease? Findings from a multisite, randomized clinical trial. Genet Med 2004;6:197-203.

12. Green RC. Risk assessment for Alzheimer's disease with genetic susceptibility testing: Has the moment arrived? Alzheimer Care Quart 2002;3:208-214.

13. Farrer LA, Cupples LA, Blackburn S, Kiely D, Auerbach S, Growdon J et al. Interrater agreement for diagnosis of Alzheimer disease: the MIRAGE study. Neurology 1994; 44:652-656.

14. Cupples LA, Risch N, Farrer LA, Myers RH. Estimation of morbid risk and age at onset with missing information. Am J Hum Genet 1991;49:76-87.

15. Lautenschlager NT, Cupples LA, Rao VS. Risk of dementia among relatives of Alz heimer's disease patients in the MIRAGE study: What is in store for the oldest old? Neurology 1996;46:641-650.

16. Sokal RR, Rohlf FJ. Biometry. New York: W.H. Freeman and Company; 1995:70.

17. Young ID. Introduction to risk calculation in genetic counseling. New York: Oxford University Press, 1991.

18. Rao CR. Linear Statistical Inference and its Applications. New York: Wiley and Sons, 1973, 382-391. 